\title{
Prospective Evaluation of Faecal Fatty Acid Excretion in Short Bowel Syndrome in Newborns
}

\author{
By Erik Heineman, Cornelis H.C. Dejong, Marjolein Piena-Spoel, Gineke Liefaard, Jan C. Molenaar, \\ and Dick Tibboel \\ Rotterdam, The Netherlands and Maastricht, The Netherlands
}

\begin{abstract}
- Short bowel syndrome (SBS) in the newborn results in limited intestinal absorptive capacity, leading especially to fatty acid (FA) malabsorption. It is unknown whether adaptation occurs in time in FA absorption, and whether this adaptation is chain-length dependent. The aim of the present study was to prospectively evaluate FA absorption and excretion during SBS in the newborn. Twenty-one neonates who underwent small bowel resection (of variable length) for various reasons (necrotizing enterocolitis, intestinal atresia, meconium peritonitis, cloacal extrophy, etc) were studied. Eight neonates had SBS, defined as a small bowel remnant of less than $50 \%$ of the original small bowel length related to gestational age. The mean remaining small bowel length in the SBS group was $34 \%(24 \%$ to $42 \%)$. The non-SBS control group consisted of 13 neonates who had only minor small bowel resections. The mean remaining bowel length for the non-SBS group was $95 \%(70 \%$ to $100 \%)$. The results show that the total fractional excretion of FA (FE-FA) at 2 weeks and $1,2,3$, and 4 months postsurgery was $51 \% \pm 37 \%$, $33 \% \pm 24 \%, 51 \% \pm 65 \%, 53 \% \pm 27 \%$, and $7 \% \pm 2 \%$ in patients with SBS, versus $12 \% \pm 8 \%, 24 \% \pm 10 \%, 9 \% \pm 3 \%$, $8 \% \pm 3 \%$ and $17 \% \pm 14 \%$ in the non-SBS controls, respectively $(\boldsymbol{P}<.05$ by ANOVA). There appeared to be an amelioration in time in FA absorption, especially in the SBS group. after 3 months. FE-FA was chain-length related, being considerably less for $\mathrm{C} 10$ and $\mathrm{C} 12$ than for $\mathrm{C} 14$ and longer amounts. An amelioration of absorption occurred in the SBS patients, especially with the longer-chain FA. On the basis of the study data, the authors conclude that in the initial adaptation phase shorter chain lengths are better absorbed than longer chain lengths; however, in the latter FA group, substantial adaptation occurs with time.

Copyright $\odot 1996$ by W.B. Saunders Company
\end{abstract}

INDEX WORDS: Short bowel syndrome, intestinal tract, adaptation, neonate, fatty acids.

CHORT BOWEL SYNDROME (SBS) occurring $D$ after massive intestinal resection is characterized by severe diarrhea, malabsorption, negative nitro-

From the Department of Paediatric Surgery, Sophia Children's Hospital, Rotterdam, The Netherlands, and the Department of Surgery, University Hospital Maastricht, Maastricht, The Netherlands.

Presented at the 42nd Annual International Congress of the British Association of Paediatric Surgeons, Sheffield, England, July 25-28, 1995.

Supported by Nutricia Nederland B.V., as a research grant for $G$. Liefaard and financial support for the laboratory investigations.

Address reprint requests to Professor Dr D. Tibboel, Department of Paediatric Surgery, Sophia Children's Hospital, Dr Molewaterplein 60, 3015 GJ Rotterdam, The Netherlands.

Copyright $\odot 1996$ by W.B. Saunders Company

0022-3468/96/3104-0012\$03.0010 gen balance, and body weight loss, primarily as a result of a drastic decrease in intestinal absorptive surface. ${ }^{1,2}$ The adaptive response in the remaining small and large intestine is marked by hyperplastic villi, with parallel increases in segmental mucosal DNA, RNA, protein, and weight. ${ }^{3-6}$ Mucosal adaptation ultimately results in diminution of malabsorptive nutritional losses. 2,7 The intestinal adaptation is achieved by the interaction of various factors for which the presence of enteral substrate is very important. ${ }^{8,9}$ Because the outcome after major bowel resection in the neonatal period depends primarily on the time needed for adaptation of the bowel, ${ }^{10}$ it is of utmost importance to achieve enteral autonomy as soon as possible. Depending on the composition of the luminal content, mucosal growth can be enhanced. ${ }^{11-15}$

Of the individual dietary components, it has been suggested that long-chain triglycerides (LCT) may be especially potent promoters of small intestinal mucosal cell proliferation. ${ }^{14,16-18}$ However, because fat malabsorption is common in these patients, ${ }^{19,20}$ supplying medium-chain triglycerides (MCT) instead of LCT is a standard practice. The reason for this is that MCT are hydrolysed more rapidly by pancreatic lipase than are $\mathbf{L C T}^{21}$ and are transported principally by the portal vein to the liver for oxidation rather than through the intestinal lymphatic vessels, which are used by LCT.22

Because little is known about the adaptation that occurs with time in MCT- and LCT-derived fatty acid (FA) malabsorption and about whether this malabsorption/adaptation is chain-length dependent, we conducted a prospective study that evaluates FA absorption and excretion in neonates with SBS and neonates who underwent minor small bowel resection.

\section{MATERIALS AND METHODS}

\section{Patients}

Twenty-one neonates had undergone intestinal resection, of variable length, as a consequence of various underlying diseases. The diagnoses were necrotizing enterocolitis (NEC), ${ }^{10}$ jejunal atresia (3), meconium peritonitis (non-cystic fibrosis) (3), cloacal extrophy (2), Hirschsprung's disease (1), multiple atresia (1), and obstruction ileus (non-cystic fibrosis) (1). Fifteen patients were born prematurely (before 37 weeks' gestation). 
The patients were divided into two groups. Eight had SBS (SBS group), defined as a loss of more than $50 \%$ of the original small bowel as related to gestational age. ${ }^{23}$ The mean remaining small bowel length related to gestational age in this group was $34 \%$ (range, $24 \%$ to $42 \%$ ). Thirteen patients had only minor small bowel resections (non-SBS group), and served as controls. Their mean remaining small bowel length related to gestational age averaged $95 \%$ (range, $70 \%$ to $100 \%$ ).

In the SBS group $(n=8)$, five patients had an ileostomy; in the non-SBS group $(n=13)$, nine patients had an ileostomy and one had a colostomy. As reported earlier, ${ }^{24}$ the intestinal continuity in these groups of patients was reestablished after an average of approximately 100 days after the initial operation. Growth was recorded regularly. Patient data are summarized in Table 1.

\section{FA Intake, Excretion, and Analysis}

The patients participated in a prospective evaluation of nutritional intake of FA and faecal excretion of FA. The protocol was begun after 2 weeks of recovery and stabilization of the patient and after informed consent was obtained from the parents or caretakers. During a 72 -hour period, enterostomy fluid or faeces were collected for analysis at week 2 and 1,2, and 3 months postoperatively, and subsequently one-time 24 -hour faeces were collected at 4,6 , and 12 months postoperatively. FA intake was calculated from the amount of food intake of a specific formula and the FA content of that formula. FA excretion was calculated from enterostomy fluid or faecal collections and from the FA concentrations. For FA analysis, the total stool sample was homogenized and the volume determined. Then, a representative sample was taken, which was analyzed by use of gas chromatography. ${ }^{25}$ Intake and excretion of FA were calculated in food and faeces (respectively) for the total of all FA and for chain lengths $\mathrm{C} 10, \mathrm{C} 12, \mathrm{C} 14, \mathrm{C} 16$, and $\mathrm{C} 18$. The fractional excretion was calculated as:

$$
100 \times \frac{\text { FA output }}{\text { FA input }}
$$

as a measure of the FA malabsorption of the gut.

\section{Statistical Analysis}

Data are presented as mean \pm SEM. $P$ values of less than .05 wese considered significant. Statistical analyses were performed with the SPSS/PC+ statistical software package on a standard personal computer. ${ }^{26}$ For analysis of the main effects of group and time as well as their interactions, analysis of variance (ANOVA) was used. For time effects within groups, the one-way ANOVA was used. Both ANOVA procedures were run for the entire study

\begin{tabular}{|c|c|c|}
\hline & SBS & NON-SBS \\
\hline No. of patients & 8 & 13 \\
\hline $\begin{array}{l}\text { Mean gestational } \\
\text { age (wk) }\end{array}$ & 35.2 (range, 28 to 40 ) & 33.7 (range, 29 to 42 ) \\
\hline Male:female ratio & $5: 3$ & $8: 5$ \\
\hline \multirow[t]{2}{*}{ Enterostomy } & 5 lleostomy & 9 lleostomy \\
\hline & & 1 Colostomy \\
\hline $\begin{array}{l}\text { Mean weight at start of } \\
\text { protocol }(\mathrm{g})\end{array}$ & $2,610 \pm 480$ & $2,500 \pm 380$ \\
\hline $\begin{array}{l}\text { Mean weight at age } \\
4 \mathrm{mo}(\mathrm{g})\end{array}$ & $3,950 \pm 490$ & $4,500 \pm 280$ \\
\hline $\begin{array}{l}\text { Mean remaining small } \\
\text { bowvel length }(\%)\end{array}$ & 34 (range, 24 to 42 ) & 95 (range, 70 to 100 ) \\
\hline
\end{tabular}

Table 2. Faecal Fatty Acid Excretlon in SBS and Non-SBS Patients

\begin{tabular}{cccccccc}
\hline \multicolumn{7}{c}{$c$} & \multicolumn{5}{c}{ No. of Months After Surgery } & & \\
\cline { 3 - 8 } & $1 / 2$ & 1 & 2 & 3 & 4 & 6 & 12 \\
\hline FE TOT & & & & & & & \\
SBS & $51 \pm 37$ & $33 \pm 24$ & $51 \pm 65$ & $53 \pm 27$ & $7 \pm 2$ & $3 \pm 0$ & 5 \\
CON & $12 \pm 8$ & $24 \pm 10$ & $9 \pm 3$ & $8 \pm 3$ & $17 \pm 14$ & 2 & \\
FE C10 & & & & & & & \\
SBS & $9 \pm 5$ & $8 \pm 4$ & $5 \pm 8$ & $15 \pm 14$ & $1 \pm 1$ & $2 \pm 1$ & 1 \\
CON & $1 \pm 1$ & $9 \pm 7$ & $0 \pm 0$ & $1 \pm 1$ & $5 \pm 5$ & 0 & \\
FE C12 & & & & & & & \\
SBS & $10 \pm 4$ & $17 \pm 18$ & 51 & $24 \pm 14$ & $2 \pm 1$ & $0 \pm 0$ & 1 \\
CON & $11 \pm 11$ & $9 \pm 8$ & 32 & $1 \pm 1$ & $10 \pm 10$ & & \\
FE C14 & & & & & & & \\
SBS & $53 \pm 75$ & 72 & 75 & 49 & $8 \pm 3$ & $2 \pm 0$ & 6 \\
CON & $17 \pm 17$ & $27 \pm 5$ & 8 & $9 \pm 6$ & $12 \pm 9$ & 2 & \\
FE C16 & & & & & & & \\
SBS & $77 \pm 50$ & $44 \pm 24$ & $61 \pm 63$ & $87 \pm 46$ & $16 \pm 4$ & $5 \pm 1$ & 14 \\
CON & $19 \pm 10$ & $38 \pm 15$ & $69 \pm 67$ & $18 \pm 10$ & $24 \pm 14$ & 6 & \\
FE C18 & & & & & & & \\
SBS & $60 \pm 37$ & $41 \pm 24$ & $58 \pm 69$ & $82 \pm 47$ & $8 \pm 3$ & $3 \pm 0$ & 2 \\
CON & $17 \pm 10$ & $37 \pm 17$ & $5 \pm 2$ & $7 \pm 2$ & $18 \pm 15$ & 1 & \\
\hline
\end{tabular}

NOTE. If the number of determinations is less than 3 , no SEM is given. Abbreviations: SBS, patients with short bowel syndrome; CON, patients with non-short bowel syndrome; FE TOT, faecal fractional excretion of total of all fatty acids; FE C10, C12, C14, C16, and C18, faecal fractional excretion of fatty acids with (respcctively) 10, 12, 14, 16 , and 18 carbon atoms.

period as well as for various shorter intervals. For clarity, only the results of the total study period and the time span between 2 weeks and 3 months postsurgery will be discussed. Differences between groups at individual time points were tested nonparametrically using the Mann Whitney U test. (Because the latter test did not yield additional valuable information, it will not be discussed further.)

\section{RESULTS}

Patient characteristics are summarized in Table 1. The SBS and non-SBS groups are comparable with the exception of the mean remaining bowel length, which was $34 \%$ (24\% to $42 \%)$ and $95 \%(70 \%$ to $100 \%$ ), respectively.

In Table 2, the fractional faecal FA excretion in SBS and non-SBS patients is shown. Data are presented as mean \pm SEM. The results show that total FA fractional excretion at 2 weeks and 1,2, 3 and 4 months postsurgery was $51 \% \pm 37 \%, 33 \% \pm 24 \%$, $51 \% \pm 65 \%, 53 \% \pm 27 \%$, and $7 \% \pm 2 \%$ in SBS patients and $12 \% \pm 8 \%, 24 \% \pm 10 \%, 9 \% \pm 3 \%$, $8 \% \pm 3 \%$, and $17 \% \pm 14 \%$ in non-SBS controls, respectively. Statistical analysis (ANOVA) for the period between 2 weeks and 3 months postsurgery showed a significant difference between the curves (Fig 1).

With respect to the fractional faecal FA excretion of the individual chain lengths, the following results were obtained. For C10 and C12 FA, the fractional 


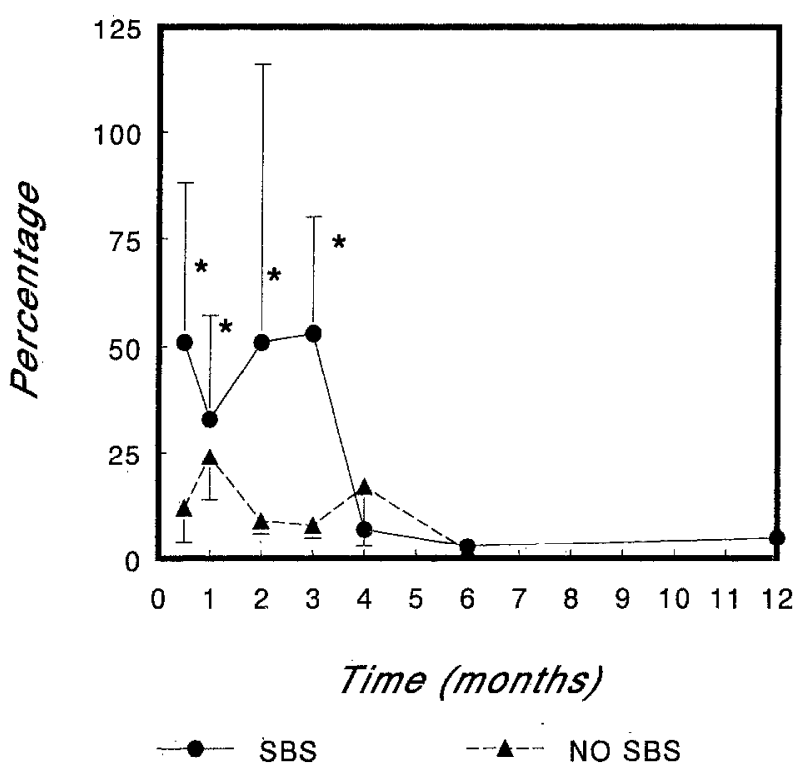

Fig 1. Fractional faecal fatty acid excretion for the total of all fatty acids in patients with short bowel syndrome $(n=8$; bowel remnant length, $34 \% \pm 3 \%$; dots) compared with patients. operated on for similar conditions but with near-normal gut remnant $(n=13$; gut remnant length, $95 \% \pm 4 \%$; triangles). Statistical significance $(P<.05$; ANOVA analysis between 2 weeks and 3 months) is indicated by the asterisks.

faecal excretion did not differ significantly for the two patient groups, and ranged, at the observed time points, between $1 \% \pm 1 \%$ and $24 \% \pm 14 \%$, with a few exceptionally high valucs. In ncither the C10 nor the $\mathrm{C} 12$ group of FA was there a significant difference between the curves (ANOVA analysis for the period between 2 weeks and 3 months as well as for all other time ranges). For the $\mathrm{C} 10 \mathrm{~F} \Lambda$, this is depicted in Fig 2. For the $\mathrm{C} 14, \mathrm{C} 16$, and $\mathrm{C} 18 \mathrm{FA}$, the fractional faecal excretion ranged from $40 \%$ to more than $80 \%$ in the SBS group, and was significantly higher (ANOVA analysis) in SBS patients in the first 3 months postsurgery. The values from 4 months postsurgery onward did not differ significantly. The curves of $\mathrm{C} 14$ and $\mathrm{C} 18$ fractional faecal FA excretion are shown in Figs 3 and 4.

\section{DISCUSSION}

In the present study we found that FA absorption (as assessed by the measurement of fractional FA excretion in stools) in neonates with SBS is dependent on FA chain length and on the time between evaluation and initial small bowel resection. The shorter chain lengths, especially $\mathrm{C} 10$ and $\mathrm{C} 12$, are absorbed almost completely from the time enteral nutrition is begun. The longer chain lengths are malabsorped for at least 12 weeks after surgery. From our data it appears that there is a separation point in

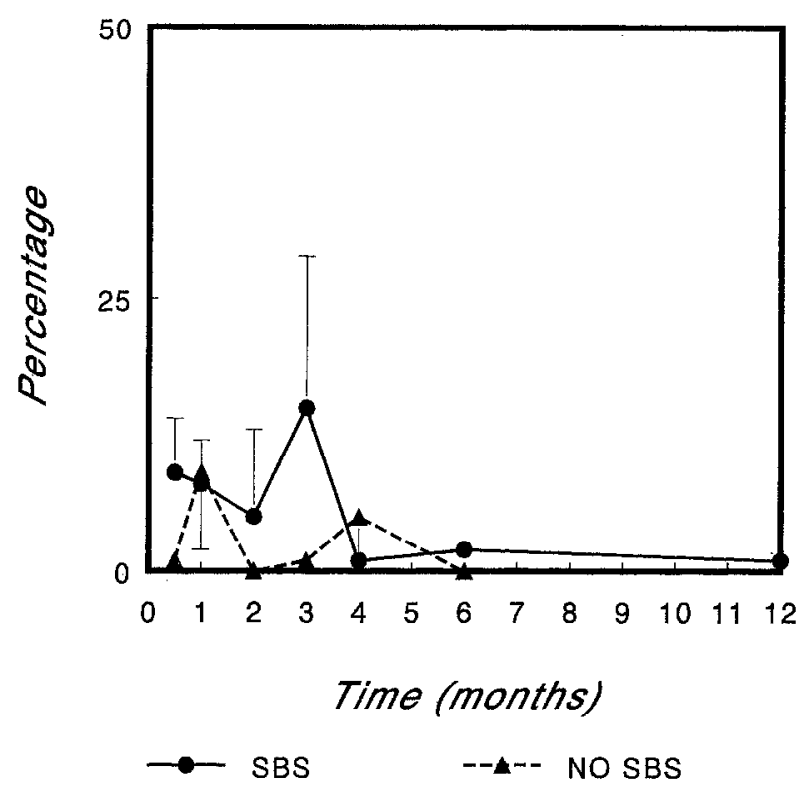

Fig 2. Fractional faecal fatty acid excretion for C10 chain length fatty acids in patients with short bowel syndrome ( $n=8$; bowel remnant length, $34 \% \pm 3 \%$; solid circles) compared with patients operated on for similar conditions but with near-normal gut remnant (n = 13; gut remnant length, $95 \% \pm 4 \%$; triangles). There were no significant differences.

the absorption in SBS neonates, between FA of up to 12 carbon atoms (MCT) and FA of 14 or more carbon atoms (LCT). The results confirm those of animal studies in which the absorption of dietary fat contain-

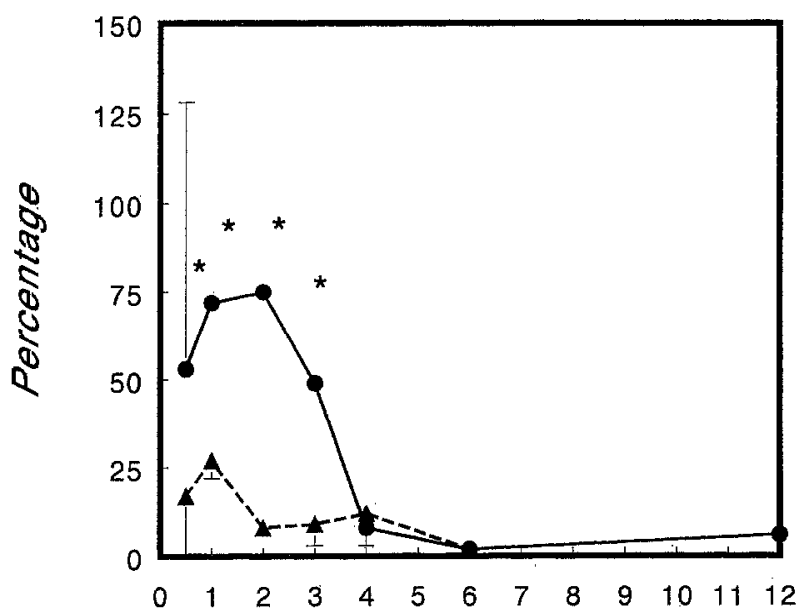

Time (months)

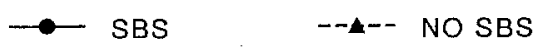

Fig 3. Fractional faecal fatty acid excretion for C14 chain length fatty acids in patients with short bowel syndrome $(n=8$; bowel remnant length, $34 \% \pm 3 \%$; dots) compared with patients operated on for similar conditions but with near-normal gut remnant $i n=13$; gut remnarit length, $95 \% \pm 4 \%$; triangles). Statistical significance ( $P<.05$; ANOVA analysis between 2 weeks and 3 months) is indicated by the asterisks. 


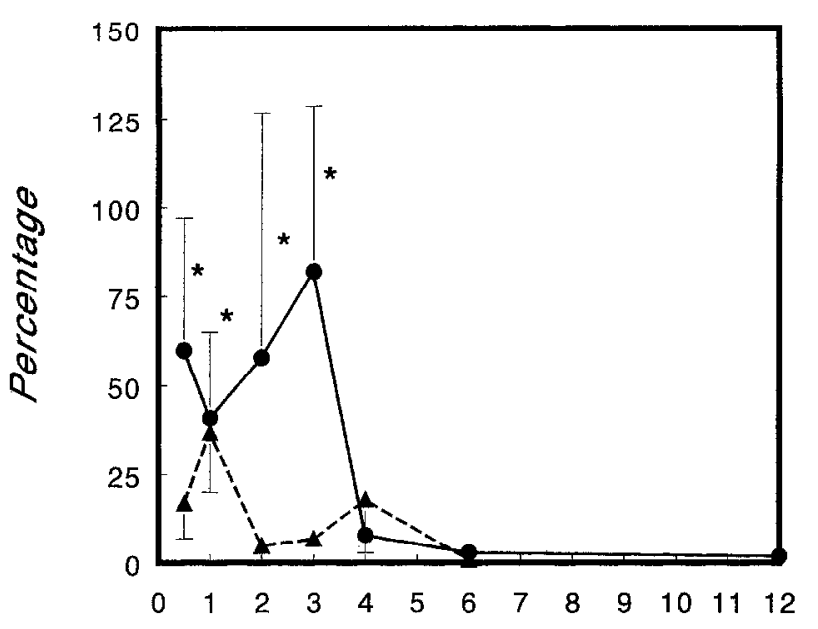

Time (months)

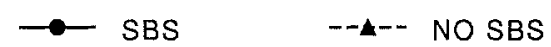

Fig 4. Fractional faecal fatty acid excretion for $\mathrm{C} 18$ chain length fatty acids in patients with short bowel syndrome $(n=8$; bowel remnant length, $34 \% \pm 3 \%$, dots) compared with patients operated on for similar conditions but with near-normal gut remnant $(n=13$; gut remnant length, $95 \% \pm 4 \%$; triangles). Statistical significance $(P<.05$; ANOVA analysis between 2 weeks and 3 months) is indicated by the asterisks.

ing LCT and MCT was evaluated and it was shown that MCT are more rapidly hydrolysed and absorbed in the intestinal lumen than are LCT. ${ }^{22}$ The proximal small bowel being the preferred side of MCT absorption and the mid and distal small bowel being the preferred side of LCT absorption might explain why there is a difference in fractional faecal FA excretion between the SBS and non-SBS groups.

Although the advantage of MCT from the standpoint of rate of absorption in SBS has been well documented, ${ }^{21,27}$ little is known about the effect of the remaining colon on FA (mal)absorption and about the effect of LCT versus MCT on mucosal adaptation.

In the present study there appeared to be a rapid decrease in the malabsorption of $\mathrm{C} 14$ and longer FA chains, and thus enhanced adaptation between 3 and 4 months postsurgery. This coincides with the closure of enterostomies around this time. ${ }^{24}$ The sudden amelioration in the absorption of the longer FA after 3 months might be attributable true adaptation in the small bowel remnant in the SBS group. Alternatively, this might be related to the inclusion of the colon in the intestinal stream after closure of enterostomies, with or without a terminal ileal segment and the ileocoecal valve. Regardless of this adaptation or its explanation, the data provide evidence that it might be better to give MCTs instead of LCTs during the first 3 months after resection.
Morin el al ${ }^{14}$ reported thal, in rats, morpholugical mucosal hyperplasia (as determined by measurement of mucosal protein and DNA content) was greater after LCT feedings than after to MCT feedings. Vanderhoof et al ${ }^{13}$ confirmed these results in rats and added the observation that mucosal function improved after the use of LCT feedings, as demonstrated by enhanced leucine uptake in the distal small bowel and enhanced sucrase activity in the proximal small bowel in the animals subjected to small bowel resection. To clarify the relative enterotrophic actions of different triglycerides, Jenkins and Thompson $^{28}$ gave rats five different oils in mixcd dicts and compared the effects on the overall mass of the small intestine and on the distribution of mass along the small intestine. Subsequently, the enterotrophic effects of bolus doses of LCT and MCT were compared. They found that LCT and MCT differed in their regional effects on cell proliferation; all four LCTrich diets increased mucosal mass and cell proliferation maximally in the mid small intestine, whereas MCT diets had the greatest effect proximally. When the LCT and MCT diets were given as boluses, the differences disappeared. The authors suggested that the enterotrophic effect of bolus MCT is the result of accelerated transit causing the release in the distal small bowel of an enterotrophic peptide that enhanced the overall trophic effect to the small bowel. It is noteworthy that the infusion of MCT directly into the human ileum causes a release of enteroglucagon, ${ }^{29}$ which has been proposed as an enterotrophic hormone. ${ }^{30}$

In summary, it is possible that LCT-rich diets have a positive effect on adaptation after șmall bowel resection. However, the issue is whether this positive LCT effect outweighs the physiological advantages of MCT. From our clinical data and the results of animal studies, it remains unclear which form of triglycerides (MCT or LCT) should be used in SBS patients. One animal study has addressed this question, comparing the relative effects of diet mixtures containing either $50 \% \mathrm{MCT} / 50 \%$ LCT or $100 \%$ LCT. ${ }^{31}$ It was shown that the diet that contained $50 \%$ MCT $/ 50 \%$ ICT led to a higher increase in the mucosal mass and protein content. It was found that the mixed diet determined the most appropriate lipid content and composition in the brush-border membrane. ${ }^{32}$ There were no deleterious effects of the mixed diet on the activities of sucrase, lactase, or aminopeptidase. ${ }^{33}$

On the basis of our data and the literature cited, it is our opinion that the enteral diet of neonates with SBS should contain a mixture of MCT and LCT, to combine the physiological advantages of MCT with 
the positive effects of LCT on adaptation and the maintenance of a sufficient supply of essential FAs (C18 provided by the LCT component). Otherwise, detrimental changes in intestinal morphology and membrane lipid composition could occur as a result of essential FA deficiency in the diet. ${ }^{34-36}$ Furthermore, avoiding enterostomies certainly enhances rehabilitation. If no reanastomosis is performed, then it should be considered to rcinfusc proximal enterostomy output in the distal enterostomy to include the remaining small bowel and colon in the adaptation process. ${ }^{35,36}$
A prospective clinical trial that compares the effects of a mixed MCT/LCT diet to those of an LCT diet in neonates with SBS is warranted. To enhance the enterotrophic effects, these diets should be given as a bolus. ${ }^{37}$

\section{ACKNOWLEDGMENT}

The authors thank Dr J.G. Bindels (Manager, Nutritional Sciences and Information) and H. van Schijndel (Business Development Manager) from Nutricia Nederland B.V., for their continuous support and criticism.

\section{REFERENCES}

1. Weser E, Fletcher JT, Urban E: Short bowel syndrome. Gastroenterology 77:572-579, 1979

2. Weser E: Nutritional aspects of malabsorption: Short gut adaptation. Clin. Gastroenterology 12:443-461, 1983

3. Dowling RH, Booth $\mathrm{CC}$ : Structural and functional changes following small intestinal resection in the rat. Clin Sci 32:139-149, 1967

4. Hanson WR: Proliferation and morphological adaptation of the intestine to experimental resection. Scand $\mathbf{J}$ Gastroenterol 17:11-20, 1982 (suppl 74)

5. Williamson RCN: Intcstinal adaptation: Structural, functional and cytokinetic changes. N Engl J Med 298:1393-1402, 1978

6. Nundy S, Malamud D, Obertop $H$, et al: Onset of cell proliferation in the shortened gut. Colonic hyperplasia after ileal resection. Gastroenterology 72:263-266, 1977

7. Bond JH, Currier BE, Buchwald M, et al: Colonic conservation of malabsorbed carbohydrates. Gastroenterology 78:444-447, 1980

8. Levine GM, Deren JJ, Yezdimir E: Small bowel resection: Oral intake is the stimulus for hyperplasia. Dig Dis Sci 21:542-546, 1976

9. Lentze MJ: Intestinal adaptation in short-bowel syndrome. Eur J Pediatr 118:291-299, 1989

10. Caniano DA, Starr I, Ginn-Pease ME: Extensive shortbowel syndrome in neonates: Outcome in the 1980s. Surgery 105:119-124, 1989

11. Spector MH, Levine GM, Deren JJ: Direct and indirect effects of dextrose and amino acids on gut mass. Gastroenterology 72:706-710, 1977

12. Buts J, Morin CL, Ling C: Influence of dietary components on intestinal adaptation after small bowel resection in rats. Clin Invest Med 2:59-66, 1979

13. Vanderhoof JA, Grandjean CA, Kaufmann SS, et al: Effect of high percentage medium-chain triglyceride diet on mucosal adaptation following massive howel resection in rats. JPEN 8:685689,1984

14. Morin CL, Grey VL, Garofalo C: Influence of lipids on intestinal adaptation after resection, in Robinson JWL, Dowling RH, Riecken EO (eds): Mechanisms of Intestinal Adaptation. Lancaster, England, MTP, 1982, pp 175-185

15. Weser E, Babbitt J, Hoban M, et al: Intestinal adaptation: Different growth responses to disaccharides compared with monosaccharides in rat small bowel. Gastroenterology 91:1521-1527, 1986

16. Weinberg LM, Pusateri JP, Levine GM: Comparison of different caloric substrates on intestinal adaptation in the rat. Gastroenterology 96:1514-1520, 1989
17. Maxton DG, Cynk EU, Jenkins AP, et al: The effect of dietary fat on the small intestinal mucosa. Gut 30:1252-1255, 1989

18. Jenkins AP, Thompson RPH: The trophic effect of Efamol on the rat small-intestinal mucosa. Clin Sci 77:555-559, 1989

19. Wapnick S, Norden DA, Venturas DJ: Essential fatty acid deficiency in patients with lesions of the gastrointestinal tract. Gut 15:367, 1974

20. Bohane TD, Haka-Tkse K, Biggar WD, et al: A clinical study of young infants after small intestinal resection. J Pediatr 94:552558,1979

21. Bach $\mathrm{AC}_{2}$ Babayan VK: Medium-chain triglycerides: $\mathrm{An}$ update. Am J Clin Nutr 36:950-962, 1982

22. Greenberger NJ, Rodgers JB, Isselbacher KJ: Absorption of medium and long-chain triglycerides: Factors influencing their hydrolysis and transport. J Clin Invest 45:217-227, 1966

23. Touloukian RJ, Walker Smith GJ: Normal intestinal length in preterm infants. J Pediatr Surg 18:720-723, 1983

24. Liefaard G, Heineman E, Molenaar JC, et al: Prospective evaluation of the absorptive capacity of the bowel after major and minor resections in the neonate. J Pedialr Surg 30:388-391, 1995

25. de Jong AEP, van den Berg TS, Nijmeijer-Couprie A, et al: Long-term aspects of phospholipidic analysis of fatty acid methy esters by use of nonpolar capillary gas chromatography. Am J Clin Nutr 57:813S, 1993 (suppl 1)

26. Norusis MJ: SPSS/PC + V 2.0 Base Manual for the IBM $\mathrm{PC} / \mathrm{XT} / \mathrm{AT}$ and PS/2. Chicago, IL, SPSS, 1988

27. Zurier RB, Campbell RG, Hashim SA, et al: Use of medium-chain triglyceride in management of patients with massive resection of the small intestine. N Engl J Med 274:490-393, 1966

28. Jenkins AP, Thompson RPH: Does the fatty acid profile of dietary fat influence its trophic effect on the small intestinal mucosa? Gut 34:358-364, 1993

29. Spiller RC, Trotman IF, Adrian TE, et al: Further characterization of the 'ileal brake' reflex in man-Effect of ileal infusion of partial digest of fat, protein and starch on jejunal motility and release of newrotensin, enternglucagon and peptide YY. Gut 29:1042-1051, 1988

30. Bloom SR, Polak JM: Enteroglucagon and the gut hormone profile of intestinal adaptation, in Robinson JWL, Dowling RH, Riecken EO (eds): Mechanisms of intestinal adaptation. Lancaster, England, MTP, 1982, pp 189-198

31. Galluser M, Czernichow B, Dreyfus H, et al: Comparison of different lipid substrates on intestinal adaptation in the rat. Gut 34:1069-1074, 1993

32. Snipes RL: The effect of essential fatty acid deficiency on the 
ultrastructure and functional capacity of the jejunal epithelium. Lab Invest 18:179-189, 1969

33. Christon R, Meskin JC, Thévenoux J, et al: Effects of a low dietary linoleic acid level on intestinal morphology and enterocyte brush border membrane lipid composition. Reprod Nutr Dev 31:691-701, 1991

34. Hart MH, Grandjean CJ, Park JHY, et al: Essential fatty acid deficiency and postresection mucosal adaptation in the rat. Gastroenterology 94:682-687, 1988

35. Puppala BL, Mangurten HH, Krant JR, et al: Distal ileos- tomy drip feedings in neonates with short bowel syndrome. J Pediatr Gastroenterol Nutr 4:489-494, 1985

36. Heineman E, Jansen M, Baeten CGMI, et al: Beneficial effects of distal ileostomy re-infusion of proximal ostomy output in neonates. Presented at the XVII Congress ESPEN, Rome, Italy, 1995 (abstr)

37. Jenkins AP, Ghatei MA, Bloom SR, et al: Effects of bolus doses of fat on small intestinal structure and on the release of gastrin, cholecystokinin, peptide tyrosine-tyrosine and enteroglucagon. Gut 33:218-223, 1992 